Research Article

\title{
The Sombor index of trees and unicyclic graphs with given maximum degree
}

\author{
Ting Zhou ${ }^{1}$, Zhen Lin $^{2, *}$, Lianying Miao ${ }^{1}$
}

${ }^{1}$ School of Mathematics, China University of Mining and Technology, Xuzhou, Jiangsu, P.R. China

${ }^{2}$ School of Mathematics and Statistics, Beijing Key Laboratory on MCAACI, Beijing Institute of Technology, Beijing, P.R. China

(Received: 5 April 2021. Received in revised form: 30 April 2021. Accepted: 3 May 2021. Published online: 5 May 2021.)

(c) 2021 the authors. This is an open access article under the CC BY (International 4.0) license (www.creativecommons.org/licenses/by/4.0/).

\begin{abstract}
Let $d_{G}(v)$ be the degree of the vertex $v$ in a graph $G$. The Sombor index of $G$ is defined as $S O(G)=\sum_{u v \in E(G)} \sqrt{d_{G}^{2}(u)+d_{G}^{2}(v)}$, which is a new vertex-degree-based topological index introduced by Gutman. Let $\mathscr{T}_{n, \Delta}$ and $\mathscr{U}_{n, \Delta}$ be the sets of trees and unicyclic graphs, respectively, with $n$ vertices and maximum degree $\Delta$. In this paper, the tree and the unicyclic graph with the minimum Sombor index from the sets $\mathscr{T}_{n, \Delta}$ and $\mathscr{U}_{n, \Delta}$, respectively, are characterized.
\end{abstract}

Keywords: Sombor index; tree; unicyclic graph; degree.

2020 Mathematics Subject Classification: 05C05, 05C07, 05C09, $05 \mathrm{C} 35$.

\section{Introduction}

Let $G$ be a simple undirected graph with vertex set $V(G)$ and edge set $E(G)$. For $v \in V(G), N_{G}(v)$ denotes the set of all neighbors of $v$, and $d_{G}(v)=\left|N_{G}(v)\right|$ denotes the degree of the vertex $v$ in $G$. Denote by $\Delta(G)$ (or $\Delta$ ) the maximum degree of $G$. A $d$-vertex of $G$ is a vertex of degree $d$. In particular, 1-vertex is called the pendant vertex or the leaf. Denote by $S_{n}$, $P_{n}$ and $C_{n}$ the star, path and cycle with $n$ vertices, respectively. Let $l\left(P_{n}\right)=\left|E\left(P_{n}\right)\right|$ and $l\left(C_{n}\right)=\left|E\left(C_{n}\right)\right|$ be the lengths of $P_{n}$ and $C_{n}$, respectively. Let $T_{n, \Delta}$, shown in Figure 1, be the tree obtained by attaching a pendant edge to each of certain $n-\Delta-1$ non-central vertices of $S_{\Delta}$, and let $U_{n, \Delta}$, shown in Figure 1, be the unicyclic graph obtained by attaching $2 \Delta-n+1$ pendant vertices and $n-\Delta-1$ paths $P_{2}$ to one vertex of the cycle $C_{3}$.

A spider is a tree with at most one vertex of degree more than two and the unique vertex of degree more than two is called the hub of the spider. A leg of a spider is a path from the hub to one of the leaves. Let $S\left(a_{1}, a_{2}, \ldots, a_{k}\right)$ be a spider with $k$ legs $P^{1}, P^{2}, \ldots, P^{k}$ for which the lengths $l\left(P^{i}\right)=a_{i}$ for $1 \leq i \leq k$. Note that $T_{n, \Delta}$ is also a spider. For convenience, denote by $T_{\Delta}$ the spider whose lengths of all $\Delta$ legs are greater than 2 (see Figure 1). Let $U_{\Delta}$ be a unicyclic graph obtained by attaching $\Delta-2$ paths of length at least 2 to a cycle (see Figure 1 ).

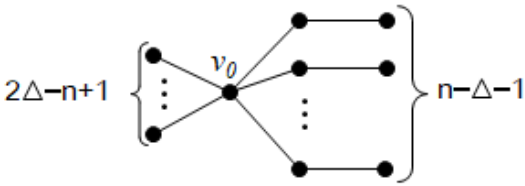

$T_{n, \Delta}$

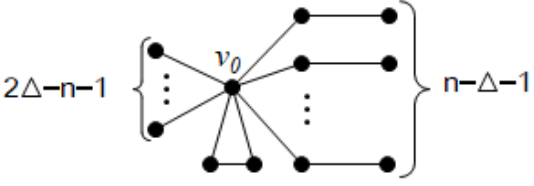

$U_{n, \Delta}$

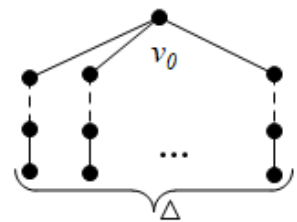

$T_{\Delta}$

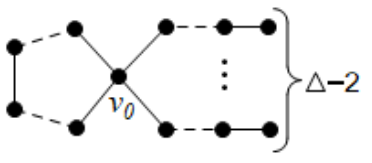

$\boldsymbol{U}_{\Delta}$

Figure 1: The graphs $T_{n, \Delta}, U_{n, \Delta}, T_{\Delta}$, and $U_{\Delta}$.

The Sombor index of a graph $G$ is defined as

$$
S O(G)=\sum_{u v \in E(G)} \sqrt{d_{G}^{2}(u)+d_{G}^{2}(v)},
$$

which is a novel vertex-degree-based topological index proposed by Gutman [6]. This new topological index immediately arises scholars' extensive attention. Deng et al. [4] showed that the Sombor index can help to predict these physico-chemical properties of octane isomers and confirmed suitability of the Sombor index in QSPR analysis. Redžepović [15] showed that the Sombor index may be used successfully on modeling thermodynamic properties of compounds due to the fact that the 
Sombor index has satisfactory prediction potential in modeling entropy and enthalpy of vaporization of alkanes. Das et al. [2] and Wang et al. [17] obtained the relations between the Sombor index and some other well-known vertex-degreebased topological index, such as the first Zagreb index, the second Zagreb index, the forgotten topological index and so on. For other related results, one may refer to $[5,7,8,10,11,14]$ and the references therein.

The extremal value problem of the topological index is of interest in graph theory and mathematical chemistry. The study of extremal value of the Sombor index of graphs has received much attention. Gutman [6] obtained extremal values of the Sombor index among the set of (connected) graphs and the set of trees. Cruz et al. [1] studied the Sombor index of chemical graphs, and characterized the graphs extremal with respect to the Sombor index over the following sets: chemical graphs, chemical trees, and hexagonal systems. Deng et al. [4] obtained a sharp upper bound for the Sombor index among all molecular trees with fixed numbers of vertices, and characterized those molecular trees achieving the extremal value. Liu [13] determined the first fourteen minimum chemical trees, the first four minimum chemical unicyclic graphs, the first three minimum chemical bicyclic graphs, the first seven minimum chemical tricyclic graphs. Réti et al. [16] characterized graphs with the maximum Sombor index in the classes of all connected unicyclic, bicyclic, tricyclic, tetracyclic, and pentacyclic graphs of a fixed order. Das et al. [3] showed that the graph constructed from the star $S_{n}$ by adding $c$ edge(s) has the maximum Sombor index among all connected $c$-cyclic graphs of order $n$ for $5 \leq c \leq n-2$, which confirms that the conjecture of Réti et al. [16] is true. Zhou et al. [18] determined the tree and the unicyclic graph with the maximum Sombor index among the set of trees and the set of unicyclic graphs with given matching number. Lin et al. [12] obtained lower and upper bounds on the spectral radius, energy and Estrada index of the Sombor matrix of graphs, and characterized the respective extremal graphs.

The purpose of this paper is to study the extremal value problem of Sombor index of trees and unicyclic graphs with given maximum degree. The following theorems are shown.

Theorem 1.1. Let $n \geq 7$ and $T \in \mathscr{T}_{n, \Delta}$, where $3 \leq \Delta \leq n-2$.

(i). If $3 \leq \Delta \leq\left\lfloor\frac{n-1}{2}\right\rfloor$, then

$$
S O(T) \geq \Delta \sqrt{\Delta^{2}+4}+2 \sqrt{2}(n-2 \Delta-1)+\sqrt{5} \Delta
$$

with equality if and only if $T \cong T_{\Delta}$.

(ii). If $\left\lfloor\frac{n-1}{2}\right\rfloor<\Delta \leq n-2$, then

$$
S O(T) \geq(n-\Delta-1) \sqrt{\Delta^{2}+4}+(2 \Delta-n+1) \sqrt{\Delta^{2}+1}+\sqrt{5}(n-\Delta-1)
$$

with equality if and only if $T \cong T_{n, \Delta}$.

Corollary 1.1. Let $T$ be a chemical tree with $n \geq 9$ vertices. If $\Delta=3$ or 4 , then

$$
S O(T) \geq 2 \sqrt{2} n+3 \sqrt{13}+3 \sqrt{5}-14 \sqrt{2} \quad \text { or } \quad S O(T) \geq 2 \sqrt{2} n+12 \sqrt{5}-18 \sqrt{2}
$$

with equality if and only if $T \cong T_{3}$ or $T \cong T_{4}$.

Theorem 1.2. Let $n \geq 5$ and $U \in \mathscr{U}_{n, \Delta}$, where $3 \leq \Delta \leq n-2$.

(i). If $3 \leq \Delta \leq\left\lfloor\frac{n+1}{2}\right\rfloor$, then

$$
S O(U) \geq \Delta \sqrt{\Delta^{2}+4}+2 \sqrt{2}(n-2 \Delta+2)+\sqrt{5}(\Delta-2)
$$

with equality if and only if $U \cong U_{\Delta}$.

(ii). If $\left\lfloor\frac{n+1}{2}\right\rfloor<\Delta \leq n-2$, then

$$
S O(U) \geq(n-\Delta+1) \sqrt{\Delta^{2}+4}+(2 \Delta-n-1) \sqrt{\Delta^{2}+1}+\sqrt{5}(n-\Delta-1)+2 \sqrt{2}
$$

with equality if and only if $U \cong U_{n, \Delta}$.

Corollary 1.2. Let $U$ be a chemical unicyclic graph with $n \geq 7$ vertices. If $\Delta=3$ or 4 , then

$$
S O(U) \geq 2 \sqrt{2} n+3 \sqrt{13}+\sqrt{5}-8 \sqrt{2} \quad \text { or } \quad S O(U) \geq 2 \sqrt{2} n+10 \sqrt{5}-12 \sqrt{2}
$$

with equality if and only if $U \cong U_{3}$ or $U \cong U_{4}$. 


\section{Preliminaries}

The distance between two vertices $u, v \in V(G)$, denoted by $d_{G}(u, v)$, is defined as the length of a shortest path between $u$ and $v$. Denote by $\bar{G}$ the complement of $G$ and by $P_{u v}$ the path between vertices $u$ and $v$. Let $G-u$ denote the graph that arises from a graph $G$ by deleting the vertex $u \in V(G)$ and all the edges incident with $u$. Let $G$ - uv denote the graph that arises from $G$ by deleting the edge $u v \in E(G)$. Similarly, $G+u v$ is the graph that arises from $G$ by adding an edge $u v \notin E(G)$, where $u, v \in V(G)$.

Lemma 2.1. [9] Let $U \subseteq \mathbb{R}$ be an open interval and $f: U \rightarrow U$ a convex function. Let $a_{1} \geq a_{2} \geq \ldots \geq a_{n}$ and $b_{1} \geq b_{2} \geq \ldots \geq$ $b_{n}$ be such elements in $U$ that inequalities $a_{1}+a_{2}+\ldots+a_{n} \geq b_{1}+b_{2}+\ldots+b_{i}$ hold for every $i \in\{1,2, \ldots, n\}$ and equality holds for $i=n$. Then

$$
f\left(a_{1}\right)+f\left(a_{2}\right)+\cdots+f\left(a_{n}\right) \geq f\left(b_{1}\right)+f\left(b_{2}\right)+\cdots+f\left(b_{n}\right) .
$$

Lemma 2.2. For $w_{0}, x_{0} \in V(G)$ (where $w_{0}, x_{0}$ are not necessarily distinct), suppose that $w_{1} w_{2} \ldots w_{k}, x_{1} x_{2} \ldots x_{h}$ are two path components in $G-w_{0}$ and $G-x_{0}$, respectively, where $k, h \geq 1$ and $w_{k}, x_{h}$ are pendant vertices of $G$. If $d_{G}\left(x_{0}\right)=s \geq 3$, $N_{G}\left(x_{0}\right)=\left\{x_{1}, v_{1}, v_{2}, \ldots, v_{s-1}\right\}, d_{G}\left(v_{i}\right) \geq 1$ for $1 \leq i \leq s-1$, let $G^{\prime}$ be a new graph with vertex set $V\left(G^{\prime}\right)=V(G)$ and edge set $E\left(G^{\prime}\right)=G-x_{0} x_{1}+w_{k} x_{1}$, see Figure 2. When $w_{0}=x_{0}, G^{\prime}$ is said to be obtained by running graph transformation $\mathbf{A}_{1}$ of $G$; when $w_{0} \neq x_{0}, G^{\prime}$ is said to be obtained by running graph transformation $\mathbf{A}_{2}$ of $G$. Then $S O(G)>S O\left(G^{\prime}\right)$.

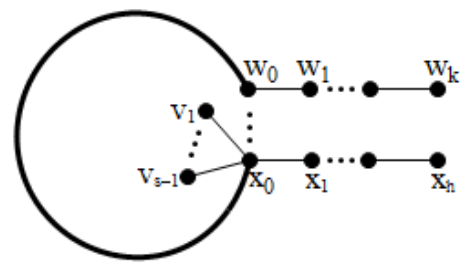

$\boldsymbol{G}$

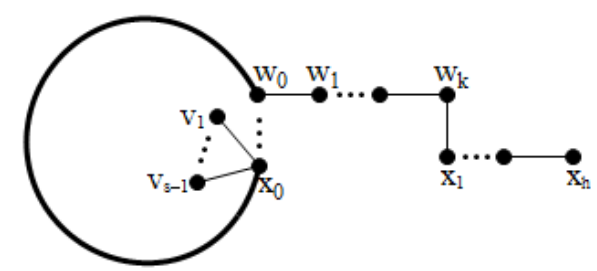

$G^{\prime}$

Figure 2: An illustration of Lemma 2.2.

Proof. From given conditions, $d_{G}\left(x_{0}\right)=s, d_{G^{\prime}}\left(x_{0}\right)=s-1, d_{G}\left(w_{k}\right)=1, d_{G^{\prime}}\left(w_{k}\right)=2$ and for any $v \in V(G) \backslash\left\{x_{0}, w_{k}\right\}$, $d_{G}(v)=d_{G^{\prime}}(v)$. Since $d_{G}\left(x_{0}\right)=s \geq 3$, by Lemma 2.1, we have

$$
\begin{aligned}
S O(G)-S O\left(G^{\prime}\right)= & \sum_{u v \in E(G)} \sqrt{d_{G}^{2}(u)+d_{G}^{2}(v)}-\sum_{u v \in E\left(G^{\prime}\right)} \sqrt{d_{G^{\prime}}^{2}(u)+d_{G^{\prime}}^{2}(v)} \\
= & \sum_{i=1}^{s-1}\left[\sqrt{d_{G}^{2}\left(x_{0}\right)+d_{G}^{2}\left(v_{i}\right)}-\sqrt{d_{G^{\prime}}^{2}\left(x_{0}\right)+d_{G}^{2}\left(v_{i}\right)}\right]+\sqrt{d_{G}^{2}\left(x_{0}\right)+d_{G}^{2}\left(x_{1}\right)} \\
& +\sqrt{d_{G}^{2}\left(w_{k-1}\right)+d_{G}^{2}\left(w_{k}\right)}-\sqrt{d_{G^{\prime}}^{2}\left(w_{k-1}\right)+d_{G^{\prime}}^{2}\left(w_{k}\right)}-\sqrt{d_{G^{\prime}}^{2}\left(w_{k}\right)+d_{G^{\prime}}^{2}\left(x_{1}\right)} .
\end{aligned}
$$

(i) If $w_{0}=x_{0}, k=h=1$, and $x_{1}=v_{s-1}$, then

$$
\begin{aligned}
S O(G)-S O\left(G^{\prime}\right) & =\sum_{i=1}^{s-2}\left[\sqrt{s^{2}+d_{G}^{2}\left(v_{i}\right)}-\sqrt{(s-1)^{2}+d_{G}^{2}\left(v_{i}\right)}\right]+2 \sqrt{s^{2}+1}-\sqrt{(s-1)^{2}+4}-\sqrt{5} \\
& >2 \sqrt{s^{2}+1}-\sqrt{(s-1)^{2}+4}-\sqrt{5} \\
& >0 .
\end{aligned}
$$

(ii) If $w_{0} \neq x_{0}$ and $k=h=1$, then

$$
\begin{aligned}
S O(G)-S O\left(G^{\prime}\right) & =\sum_{i=1}^{s-1}\left[\sqrt{s^{2}+d_{G}^{2}\left(v_{i}\right)}-\sqrt{(s-1)^{2}+d_{G}^{2}\left(v_{i}\right)}\right]+\sqrt{s^{2}+1}+\sqrt{d_{G}^{2}\left(w_{0}\right)+1}-\sqrt{d_{G}^{2}\left(w_{0}\right)+4}-\sqrt{5} \\
& >\sqrt{s^{2}+1}+\sqrt{d_{G}^{2}\left(w_{0}\right)+1}-\sqrt{d_{G}^{2}\left(w_{0}\right)+4}-\sqrt{5} \\
& >0 .
\end{aligned}
$$

(iii) If $k \geq 2, h=1$, then

$$
S O(G)-S O\left(G^{\prime}\right)=\sum_{i=1}^{s-1}\left[\sqrt{s^{2}+d_{G}^{2}\left(v_{i}\right)}-\sqrt{(s-1)^{2}+d_{G}^{2}\left(v_{i}\right)}\right]+\sqrt{s^{2}+1}+\sqrt{5}-\sqrt{8}-\sqrt{5}>\sqrt{s^{2}+1}-\sqrt{8}>0 .
$$


(iv) If $k, h \geq 2$, then

$$
\begin{aligned}
S O(G)-S O\left(G^{\prime}\right) & =\sum_{i=1}^{s-1}\left[\sqrt{s^{2}+d_{G}^{2}\left(v_{i}\right)}-\sqrt{(s-1)^{2}+d_{G}^{2}\left(v_{i}\right)}\right]+\sqrt{s^{2}+4}+\sqrt{5}-2 \sqrt{8} \\
& >\sqrt{s^{2}+4}+\sqrt{5}-2 \sqrt{8} \\
& >\sqrt{13}+\sqrt{5}-2 \sqrt{8} \\
& >0 .
\end{aligned}
$$

Combining the above arguments, we have the proof.

\section{The proof of Theorem 1.1}

In this section, we determine the tree with the minimum Sombor index among trees with $n$ vertices and maximum degree $\Delta$. If $\Delta=2$, then $\mathscr{T}_{n, \Delta}=\left\{P_{n}\right\}$. If $\Delta=n-1$, then $\mathscr{T}_{n, \Delta}=\left\{S_{n}\right\}$. From now on, we assume that $3 \leq \Delta \leq n-2$.

Proof of Theorem 1.1. Let $T$ be a tree with $n$ vertices and maximum degree $\Delta$ that minimize the Sombor index and let $v_{0}$ be a $\Delta$-vertex of $T$. We will show the following Claims 1-3, which, put together, will get our proof.

Claim 1. $T$ is a spider.

Proof. Suppose $T$ is not a spider. There exists $v \in V(T) \backslash\left\{v_{0}\right\}$, such that $d_{T}(v) \geq 3$. Then we can get a new tree $T_{1} \in \mathscr{T}_{n, \Delta}$ by running graph transformation $\mathbf{A}_{1}$ on $v$. By Lemma 2.2, $S O\left(T_{1}\right)<S O(T)$, which contradicts the choice of $T$. Thus $T$ is a spider.

Let $T=S\left(a_{1}, a_{2}, \ldots, a_{\Delta}\right)$ with $\Delta$ legs $P^{1}, P^{2}, \ldots, P^{\Delta}$, and the lengths $l\left(P^{i}\right)=a_{i}$ for $1 \leq i \leq \Delta$. Without loss of generality, we assume that $a_{1} \geq a_{2} \geq \ldots \geq a_{\Delta}$.

Claim 2. If $3 \leq \Delta \leq\left\lfloor\frac{n-1}{2}\right\rfloor$, then $a_{\Delta} \geq 2$.

Proof. Suppose $a_{\Delta}=1$. Since $n-1 \geq 2 \Delta$, we have $a_{1} \geq 3$. Let $P^{1}=v_{0} v_{1}^{1} v_{2}^{1} \ldots v_{s}^{1}$ where $s \geq 3$ and $P_{\Delta}=v_{0} v_{\Delta}$. Let $T_{2}=T-v_{s-1}^{1} v_{s}^{1}+v_{\Delta} v_{s}^{1}$, by Lemma 2.2 , we have $S O(T)-S O\left(T_{2}\right)=\sqrt{\Delta^{2}+1}+\sqrt{8}-\sqrt{\Delta^{2}+4}-\sqrt{5}>0$, which contradicts the choice of $T$. Thus $a_{\Delta} \geq 2$, that is, $T \cong T_{\Delta}$.

Claim 3. If $\left\lfloor\frac{n-1}{2}\right\rfloor<\Delta \leq n-2$, then $a_{1} \leq 2$.

Proof. Suppose $a_{1} \geq 3$. Since $n-1<2 \Delta$, we have $a_{\Delta}=1$. Let $P^{1}=v_{0} v_{1}^{1} v_{2}^{1} \ldots v_{s}^{1}$ where $s \geq 3$ and $P_{\Delta}=v_{0} v_{\Delta}$. Similar to the proof of Claim 2, let $T_{3}=T-v_{s-1}^{1} v_{s}^{1}+v_{\Delta} v_{s}^{1}$, then $S O(T)>S O\left(T_{3}\right)$, which contradicts the choice of $T$. Thus $a_{1} \leq 2$, that is, $T \cong T_{n, \Delta}$.

By direct calculations, we get $S O\left(T_{\Delta}\right)=\Delta \sqrt{\Delta^{2}+4}+\sqrt{8}(n-2 \Delta-1)+\sqrt{5} \Delta$ and

$$
S O\left(T_{n, \Delta}\right)=(n-\Delta-1) \sqrt{\Delta^{2}+4}+(2 \Delta-n+1) \sqrt{\Delta^{2}+1}+\sqrt{5}(n-\Delta-1) .
$$

This completes the proof of Theorem 1.1.

\section{The proof of Theorem 1.2}

In this section, we determine the unicyclic graph with the minimum Sombor index among unicyclic graphs with $n$ vertices and maximum degree $\Delta$. If $\Delta=2$, then $\mathscr{U}_{n, \Delta}=\left\{C_{n}\right\}$. If $\Delta=n-1$, then $\mathscr{U}_{n, \Delta}=\left\{S_{n}+e\right\}$, where $e$ is a edge in $\bar{S}_{n}$. Next, we assume that $3 \leq \Delta \leq n-2$.

Proof of Theorem 1.2. Let $U$ be a unicyclic graph with $n$ vertices and maximum degree $\Delta$ that minimize the Sombor index. Suppose $C$ is the unique cycle of $U$. If there exist a $\Delta$-vertex on $C$, then we chose it and denote by $v_{0}$; otherwise, chose any $\Delta$-vertex, also denote by $v_{0}$. First, we assume that $v_{0} \notin V(C)$. Then there is a vertex $v \in V(C)$ such that $d_{U}\left(v, v_{0}\right)=\min \left\{d_{U}\left(u, v_{0}\right) \mid u \in V(C)\right\}$, clearly, $d_{U}(v) \geq 3$. We will show the following Claims 1-5, which, put together, will get our proof.

Claim 1. For any $u \in V(U) \backslash\left\{v_{0}, v\right\}, d_{U}(u) \leq 2$. 
Proof. If the claim is not true, there are three cases:

Case i. there exists $u \in V(U) \backslash\left(V(C) \cup P_{v v_{0}}\right)$ such that $d_{U}(u) \geq 3$. Then we can get a new unicyclic graph $U_{1} \in \mathscr{U}_{n, \Delta}$ by running graph transformation $\mathbf{A}_{1}$ on $u$. By Lemma 2.2, $S O(U)>S O\left(U_{1}\right)$, which contradicts the choice of $U$.

Case ii. there exists $u \in\left(V(C) \cup P_{v v_{0}}\right) \backslash\left\{v, v_{0}\right\}$ such that $d_{U}(u) \geq 4$. There are at least two paths starting from $u$ to pendant vertices of $U$, then by running transformation $\mathbf{A}_{1}$ on $u$, we can get a contradiction.

Case iii. there exists $u \in\left(V(C) \cup P_{v v_{0}}\right) \backslash\left\{v, v_{0}\right\}$ such that $d_{U}(u)=3$. Let $u u_{1} u_{2} \ldots u_{s}$ be the path from $u$ to pendant vertex $u_{s}$, where $s \geq 1$ and $d_{U}\left(u_{i}\right)=2$ for $1 \leq i \leq s-1$. And let $v_{0} v_{1} v_{2} \ldots v_{t}$ be one of the paths from $v_{0}$ to a pendant vertex $v_{t}$, where $t \geq 1$ and $d_{U}\left(v_{i}\right)=2$ for $1 \leq i \leq t-1$. Let $U_{2}=U-u u_{1}+v_{s} u_{1}$, then $U_{2}$ is obtained by running transformation $\mathbf{A}_{2}$ from $U$ and $U_{2} \in \mathscr{U}_{n, \Delta}$. By Lemma 2.2, we have $S O(U)>S O\left(U_{2}\right)$, which contradicts the choice of $U$.

Combining the above cases, we have $d_{U}(u) \leq 2$ for any $u \in V(U) \backslash\left\{v_{0}, v\right\}$.

Claim 2. $d_{U}(v) \leq 3$.

Proof. If $d_{U}(v) \geq 5$, there are at least two paths starting from $v$ to pendant vertices of $U$, similarly, by running transformation $\mathbf{A}_{1}$, we can get a contradiction. If $d_{U}(v)=4$, by running transformation $\mathbf{A}_{2}$, we can also get a contradiction.

Denote by $v_{1}, v_{2}, \ldots, v_{\Delta}$ the neighbors of $v_{0}$, where $v_{1} \in V\left(P_{v v_{0}}\right)$.

Claim 3. $v=v_{0}$, that is to say, there must be $v_{0} \in V(C)$.

Proof. For otherwise, we can get a new unicyclic graph $U_{3}=U-\left\{v_{0} v_{i} \mid 2 \leq i \leq \Delta-2\right\}+\left\{v v_{i} \mid 2 \leq i \leq \Delta-2\right\}$ and $U_{3} \in \mathscr{U}_{n, \Delta}$. For $\Delta-1 \leq i \leq \Delta, d_{U}\left(v_{i}\right) \leq 2$, we have

$$
\begin{aligned}
S O(U)-S O\left(U_{3}\right) & =2 \sqrt{3^{2}+2^{2}}+\sum_{i=\Delta-1}^{\Delta} \sqrt{\Delta^{2}+d_{U}^{2}\left(v_{i}\right)}-2 \sqrt{\Delta^{2}+2^{2}}-\sum_{i=\Delta-1}^{\Delta} \sqrt{3^{2}+d_{U}^{2}\left(v_{i}\right)} \\
& =\left[2 \sqrt{3^{2}+2^{2}}-\sum_{i=\Delta-1}^{\Delta} \sqrt{3^{2}+d_{U}^{2}\left(v_{i}\right)}\right]-\left[2 \sqrt{\Delta^{2}+2^{2}}-\sum_{i=\Delta-1}^{\Delta} \sqrt{\Delta^{2}+d_{U}^{2}\left(v_{i}\right)}\right] \\
& \geq 0 .
\end{aligned}
$$

We have now in $U_{3}, d_{U_{3}}(v)=\Delta$ and $d_{U_{3}}\left(v_{0}\right)=3$, then there are at least two paths starting from $v_{0}$ to pendant vertices of $U_{3}$, similarly, by running transformation $\mathbf{A}_{1}$ on $v_{0}$, we can get a contradiction, which contradicts the choice of $U$. Thus $v_{0} \in V(C)$.

By Claims 1-3, $U$ is a unicyclic graph obtained by attaching $\Delta-2$ paths to the vertex $v_{0}$ of cycle $C$. Let $v_{1}, v_{2} \in V(C)$. Similar to the proof of Theorem 1.1, denote by $P^{i}$ the path from $v_{0}$ to a pendant vertex of $U$ and $v_{i} \in P^{i}$, where $3 \leq i \leq \Delta$. Without loss of generality, we can assume that $l\left(P^{3}\right) \geq l\left(P^{4}\right) \geq \ldots \geq l\left(P^{\Delta}\right)$.

Claim 4. If $3 \leq \Delta \leq\left\lfloor\frac{n+1}{2}\right\rfloor$, then $l\left(P^{\Delta}\right) \geq 2$.

Proof. Suppose that $l\left(P^{\Delta}\right)=1$. Since $n-3 \geq 2(\Delta-2)$, we have the following two cases:

Case i. $l\left(P^{3}\right) \geq 3$. Let $P^{\Delta}=v_{0} v_{\Delta}$ and $P^{3}=v_{0} v_{3} v_{2}^{3} \ldots v_{s}^{3}$, where $s \geq 3$. Let $U_{4}=U-v_{s-1}^{3} v_{s}^{3}+v^{\Delta} v_{s}^{3}$, by Lemma 2.2, we have $S O(U)-S O\left(U_{4}\right)=\sqrt{\Delta^{2}+1}+\sqrt{8}-\sqrt{\Delta^{2}+4}-\sqrt{5}>0$, which contradicts the choice of $U$.

Case ii. $l\left(P^{3}\right)=2$ and $|E(C)| \geq 4$. Let $x, y, z$ be three vertices on $C$ different from $v_{0}$ such that $x y, y z \in E(C)$. Let $U_{5}=U-x y-y z+x z+v_{\Delta} y$, we have $S O(U)-S O\left(U_{5}\right)=\sqrt{\Delta^{2}+1}+\sqrt{8}-\sqrt{\Delta^{2}+4}-\sqrt{5}>0$, which contradicts the choice of $U$.

Thus, $l\left(P^{\Delta}\right) \geq 2$, that is, $U \cong U_{\Delta}$.

Claim 5. If $\left\lfloor\frac{n+1}{2}\right\rfloor<\Delta \leq n-2$, then $l\left(P^{3}\right) \leq 2$ and $|E(C)|=3$.

Proof. Note that $n-3<2(\Delta-2)$. If $l\left(P^{3}\right) \geq 3$ or $|E(C)| \geq 4$, then $l\left(P^{\Delta}\right)=1$. Similar to the proof of Claim 4 , we can get a contradiction. Thus $l\left(P^{3}\right) \leq 2$ and $|E(C)|=3$, that is, $U \cong U_{n, \Delta}$.

By direct calculations, we get $S O\left(U_{\Delta}\right)=\Delta \sqrt{\Delta^{2}+4}+\sqrt{8}(n-2 \Delta+2)+\sqrt{5}(\Delta-2)$ and

$$
S O\left(U_{n, \Delta}\right)=(n-\Delta+1) \sqrt{\Delta^{2}+4}+(2 \Delta-n-1) \sqrt{\Delta^{2}+1}+\sqrt{5}(n-\Delta-1)+\sqrt{8} .
$$

This completes the proof of Theorem 1.2. 


\section{Acknowledgments}

This research is supported by the National Natural Science Foundation of China (through grant no. 11771443). The authors are grateful to the anonymous referees for careful reading and valuable comments which result in an improvement of the original manuscript.

\section{References}

[1] R. Cruz, I. Gutman, J. Rada, Sombor index of chemical graphs, Appl. Math. Comput. 399 (2021) Art\#126018.

[2] K. C. Das, A. S. Çevik, I. N. Cangul, Y. Shang, On Sombor index, Symmetry 13 (2021) Art\# 140.

[3] K. C. Das, A. Ghalavand, A.R. Ashrafi, On a conjecture about the Sombor Index of graphs, arXiv:2103.17147 [math.CO], (2021).

[4] H. Deng, Z. Tang, R. Wu, Molecular trees with extremal values of Sombor indices, Int. J. Quantum Chem., DOI: 10.1002/qua.26622, In press.

[5] T. Došlić, T. Réti, A. Ali, On the structure of graphs with integer Sombor indices, Discrete Math. Lett. 7 (2021) 1-4.

[6] I. Gutman, Geometric approach to degree-based topological indices: Sombor indices, MATCH Commun. Math. Comput. Chem. 86 (2021) 11-16.

[7] I. Gutman, Some basic properties of Sombor indices, Open J. Discrete Appl. Math. 4 (2021) 1-3.

[8] N. Ghanbari, S. Alikhan, Sombor index of certain graphs, arXiv:2102.10409 [math.CO], (2020).

[9] J. Karamata, Sur une inégalité relative aux fonctions convexes, Publ. Math. Univ. Belgrade 1 (1932) 145-148.

[10] V. R. Kulli, Sombor indices of certain graph operators, Int. J. Eng. Sci. Res. Tech. 10 (2021) 127-134.

[11] V. R. Kulli, I. Gutman, Computation of Sombor Indices of certain networks, Int. J. Appl. Chem. 8 (2021) 1-5.

[12] Z. Lin, L. Miao, T. Zhou, On the spectral radius, energy and Estrada index of the Sombor matrix of graphs, arXiv:2102.03960 [math.CO], (2021).

[13] H. Liu, Ordering chemical graphs by their Sombor indices, arXiv:2103.05995 [math.CO], (2021).

[14] I. Milovanović, E. Milovanović, M. Matejić, On some mathematical properties of Sombor indices, Bull. Int. Math. Virtual Inst. 11 (2021) $341-353$.

[15] I. Redžepović, Chemical applicability of Sombor indices, J. Serb. Chem. Soc., DOI: 10.2298/JSC201215006R, In press.

[16] T. Réti, T. Došlić, A. Ali, On the Sombor index of graphs, Contrib. Math. 3 (2021) 11-18.

[17] Z. Wang, Y. Mao, Y. Li, B. Furtula, On relations between Sombor and other degree-based indices, J. Appl. Math. Comput., DOI: 10.1007/s12190021-01516-x, In press.

[18] T. Zhou, Z. Lin, L. Miao, The Sombor index of trees and unicyclic graphs with given matching number, arXiv:2103.04645 [math.CO], (2021). 